\title{
Static and dynamic tensile behaviour of aluminium processed by high pressure torsion
}

\author{
Patricia Verleysen ${ }^{1, \text { a }}$, Wouter Oelbrandt ${ }^{1}$, Soroosh Naghdy $^{1}$, and Leo Kestens ${ }^{1,2}$ \\ ${ }^{1}$ Ghent University, Faculty of Engineering and Architecture, Department of Materials Science and Engineering, \\ Technologiepark 903, 9052 Zwijnaarde \\ ${ }^{2}$ Delft University of Technology, Faculty of 3mE, PO Box 5025, 2600 GA Delft, The Netherlands
}

\begin{abstract}
High pressure torsion (HPT) is a severe plastic deformation technique in which a small, disk-like sample is subjected to a torsional deformation under a high hydrostatic pressure. In present study, the static and dynamic tensile behaviour of commercially pure aluminium $(99.6 \mathrm{wt} \%)$ processed by HPT is studied. The high strain rate tensile behaviour is characterized using a purpose-developed miniature split Hopkinson tensile bar setup by which strain rates up to $5 \times 10^{3} \mathrm{~s}^{-1}$ can be reached. During the tests, the deformation of a speckle pattern applied to the samples is recorded, by which local information on the strain is obtained using a digital image correlation technique. Electron back scatter diffraction images are used to investigate the microstructural evolution, more specifically the grain refinement obtained by HPT. The fracture surfaces of the tensile samples are studied by scanning electron microscopy. Results show that the imposed severe plastic deformation significantly increases the tensile strength, however, at the expense of ductility. The strain rate only has a minor influence on the materials tensile behaviour.
\end{abstract}

\section{Introduction}

Processing of metals using severe plastic deformation (SPD) is now a well-established procedure for refining the grain size of bulk samples to the submicrometer or nanometer level. Among different SPD approaches, equal channel angular pressing (ECAP) [1], high pressure torsion $[2,3]$ and accumulated roll bonding (ARB) [4] have been mostly applied. In high pressure torsion (HPT) processes a small, thin disk-like sample is subjected to a torsional deformation under high hydrostatic pressure. Neglecting thinning of the sample, a shear strain is obtained which linearly evolves as a function of the distance to the centre of the sample.

The strain-induced grain refinement gives rise to materials with exceptional properties. Strength increases typically with a factor of 5 have been reported in literature [5]. Grain refinement is the main strengthening mechanism for the thus-obtained nanocrystalline (NC, grain size smaller than $100 \mathrm{~nm}$ ), ultrafine grained (UFG, $\mathrm{d} \sim 200 \mathrm{~nm}$ ) or microcrystalline (MC, $\mathrm{d}>1$ micron) metals, which follow in general the well-known Hall-Petch relation which relates the strength of polycrystalline materials with the grain size [6].

Next to a higher strength, for some metals, significant changes in strain rate sensitivity (SRS) are reported. Independent of the processing route used to refine the grain size, the SRS of face-centered cubic metals is found to be higher whereas for body-centered cubic metals a reduced SRS is observed compared to their coarsegrained counterparts [7]. An increased SRS contributes to the tensile ductility of materials which is most

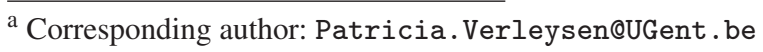

often low for fine-grained materials. Indeed, although reasonable fracture elongations are commonly obtained, major drawback of fine-grained materials is that they suffer from low uniform strains in tensile tests. This is mainly attributed to the fact that the size of SPD evolved grains is too small to allow for efficient storage of dislocations and further strain hardening [8]. An increased strain rate sensitivity is proposed as one of the strategies to achieve higher uniform tensile deformation in a nanostructured metal. Strain rate sensitivity is dependent on the deformation mechanisms and, as such, dependent on the strain rate [7].

The $\mathrm{m}$-value is determined using strain rate jump experiments or experiments carried out at different strain rates $[7,9]$. The reported strain rates during the tests are all in the low, quasi-static strain rate regime $(\leq 10-1 / \mathrm{s})$. To the authors knowledge no information can be found on the high strain rate tensile behaviour of fine-grained materials. Characterisation of the mechanical behaviour of HPT processed samples is not obvious, because of the intrinsic heterogeneity and small size of the samples. Both necessitate a local measurement approach for the strain. At high strain rates, the short test duration and the generally reduced deformation capacity of the sample, make accurate characterization of the tensile response even more difficult.

In present work, static and dynamic tensile experiments are performed on commercially pure aluminium (99.6 wt\%) processed by HPT. From small disks subjected to several levels of deformation by HPT, miniature dogbone tensile specimens are extracted. The equivalent strain achieved by HPT in the gage section varies from 0 to 32. The resulting grain refinement is assessed by EBSD measurements. Static tensile tests are carried out 
Table 1. Chemical composition of the pure aluminium.

\begin{tabular}{cccccc}
$\mathrm{Fe}$ & $\mathrm{Si}$ & $\mathrm{Cu}$ & $\mathrm{Mg}$ & $\mathrm{Mn}$ & $\mathrm{Al}$ \\
\hline 0.280 & 0.050 & 0.049 & 0.018 & 0.005 & 99.6
\end{tabular}

at room temperature at a strain rate of $2.8 \times 10^{-3} \mathrm{~s}^{-1}$. To obtain local information on the strain, a speckle pattern applied on the samples is recorded during deformation and processed by a Digital Image Correlation (DIC) technique. To study the high strain rate tensile behaviour, a miniature split Hopkinson tensile bar setup is developed by which strain rates up to $4.4 \times 10^{3} \mathrm{~s}^{-1}$ can be reached. Fracture surfaces of the static and dynamic tensile samples are observed using Scanning Electron Microscopy (SEM).

\section{Materials and experiments}

\subsection{Material}

The tested material is a commercial purity aluminium with a chemical composition given in Table 1. The material was rolled to a plate with an initial thickness of $1.1 \mathrm{~mm}$. From the rolled plate, disks with a diameter of $15 \mathrm{~mm}$ were punched. The disks were annealed for $1 \mathrm{~h}$ in air at $823 \mathrm{~K}$ to reach an initial grain size of approximately $300 \mu \mathrm{m}$.

The disks were subjected to a rotational deformation of $1 / 8,1 / 4,1 / 2,1,2$ and 3 rotations in a HPT device under a nominal pressure of $2.5 \mathrm{GPa}$. The anvils of the device are designed to ensure semi-confined deformation conditions [2]. To estimate the equivalent strain during torsion the von Mises strain is widely used [2]:

$$
\varepsilon_{\nu M}=\gamma / \sqrt{3} .
$$

In this equation $\gamma$ is the engineering shear strain. Assuming that the thickness $h$ of the disk is independent of the rotation angle $\theta$ and does not change significantly during rotation, $\gamma$ can be calculated at a radial distance $r$ from the centre by $\lambda=\mathrm{r} \theta / \mathrm{h}$.

\subsection{Static and dynamic tensile test techniques}

The experiments at low strain rates are carried out on a $5 \mathrm{kN}$ Deben $^{\mathrm{TM}}$ microtensile test device at TUDelft. Miniature dogbone shaped samples with a gage length of $3 \mathrm{~mm}$ and width of $1 \mathrm{~mm}$ were extracted from the HPT processed aluminium disks by electro discharge machining. The centre of the gage length is at a distance of $3 \mathrm{~mm}$ from the centre of the disk, see Fig. 1. In Table 2 the equivalent von Mises strain in the centre of the samples is given calculated using Eq. (1). The samples are mounted horizontally. The tests are performed at room temperature with a crosshead velocity of $8.3 \cdot 10^{-3} \mathrm{~mm} / \mathrm{s}$.

The clamp displacement and force are recorded during testing, from which respectively the average strain and stress in the gage section of the sample are calculated. To obtain reliable local strain data a speckle pattern is applied to the sample surface prior to testing. During a test, the deforming speckle pattern is recorded using an Olympus SZX9 light optical microscope equipped with a camera, see Fig. 2. After testing the images are processed using a Digital Image Correlation (DIC) technique to calculate the local strain field.

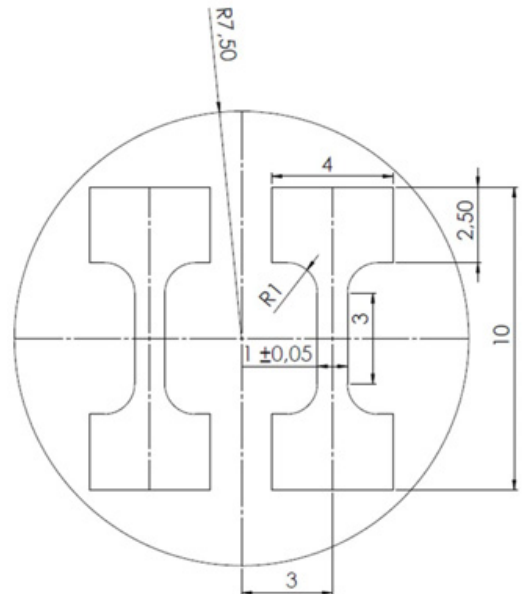

Figure 1. Miniature tensile specimens extracted from HPT processed aluminium disks with a diameter of $15 \mathrm{~mm}$.

Table 2. Equivalent strain in the centre of the gage section of the samples calculated using Eq. (2), grain size measured by EBSD, number of grains a cross section of gage section of the tensile samples and dimple size observed in SEM images of the fracture surface of the statically loaded samples.

\begin{tabular}{|c|c|c|c|}
\hline $\begin{array}{c}\text { Applied } \\
\text { rotations }\end{array}$ & $\begin{array}{c}\text { Equivalent } \\
\text { strain }\end{array}$ & $\begin{array}{c}\text { Measured } \\
\text { grain size }\end{array}$ & $\begin{array}{c}\text { Dimple } \\
\text { size } \\
\text { fracture }\end{array}$ \\
\hline$[$ rot] & {$[-]$} & {$[$ micron] } & [micron] \\
\hline 0 & 0 & 300.12 & 12.04 \\
\hline $1 / 8$ & 1.36 & 184.76 & 13.42 \\
\hline $1 / 4$ & 2.72 & 103.39 & 12.93 \\
\hline $1 / 2$ & 5.44 & 3.37 & 9.87 \\
\hline 1 & 10.88 & 2.42 & 8.47 \\
\hline 2 & 21.77 & 1.04 & 9.67 \\
\hline 3 & 32.65 & 1.05 & 8.37 \\
\hline
\end{tabular}

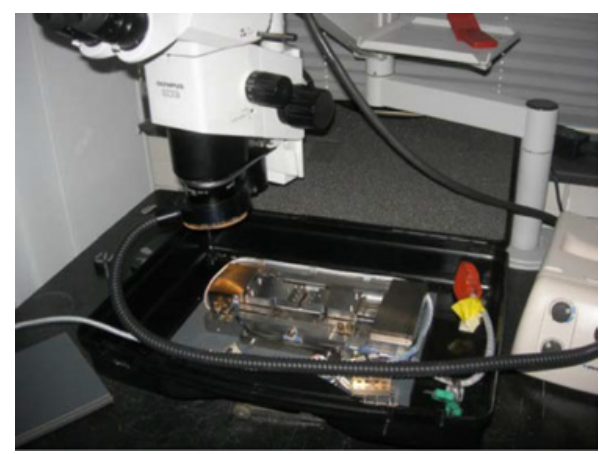

Figure 2. Microtensile test device equipped with a light optical microscope used for the static tensile tests.

High strain rate tensile test were carried out using a purpose-developed Split Hopkinson Tensile Bar (SHTB) setup. The setup consists of two Hopkinson bars - an input and output bar - between which the sample is fixed, see Fig. 3. A tube-like impactor is put around the input bar. To perform a test, the impactor is accelerated towards an anvil at the free end of the input bar, thus generating an 


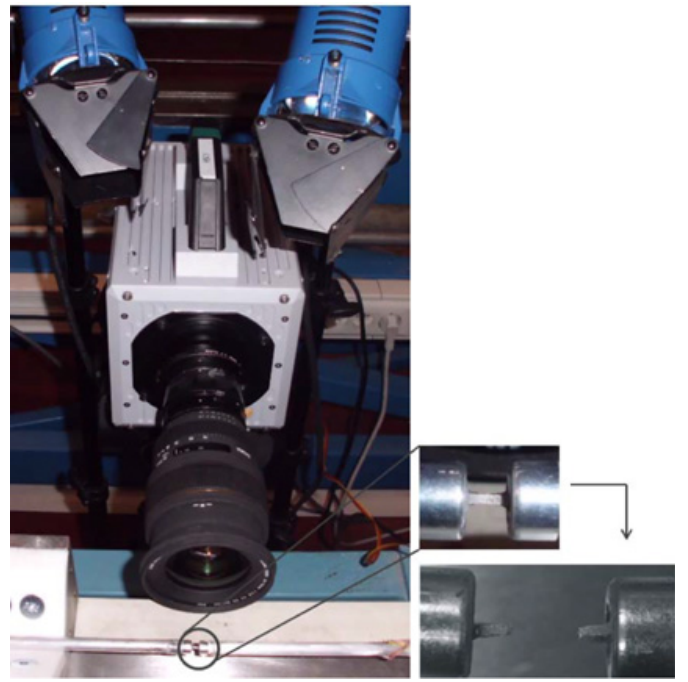

Figure 3. Tensile specimen glued between the Hopkinson bars together with high speed camera used to record the specimen deformation (left), before and after testing (right).
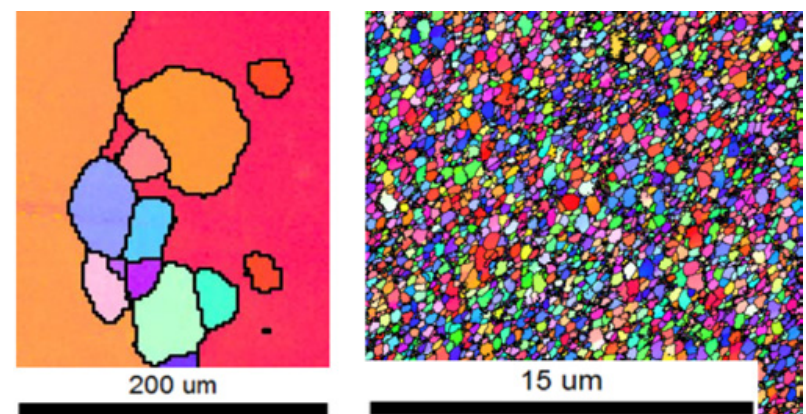

Figure 4. EBSD images of the undeformed material with a mean grain size of 300 micron (left) and the material deformed up to an equivalent strain of 50 with a mean grain size of 1.01 micron (right).

incident tensile wave with a length of $1 \mathrm{~m}$ and an amplitude proportional with the impactor velocity. In order to limit the impedance mismatch between the Hopkinson bars and the sample, and to capture the low force needed to deform the sample with a sufficiently high accuracy, the input and output bars consist of aluminium tubes with lengths of $2.1 \mathrm{~m}$ and $1.2 \mathrm{~m}$ respectively, and outer diameter of $8 \mathrm{~mm}$ and wall thickness of $1 \mathrm{~mm}$.

\section{Results}

\subsection{Grain refinement obtained by HPT}

The grain size of the HPT processed samples is determined by electron back scatter diffraction (EBSD). In Fig. 4 EBSD images are represented of the annealed aluminium and a material sample in which the saturation grain size is reached (approximately after an equivalent strain of 15). Comparison of the two images clearly shows the obvious grain refinement obtained by the SPD process. In Table 2 values of the grain size are given at a distance of $3 \mathrm{~mm}$ away from the centre the HPT disk samples. This location corresponds with the centre of the gage section of the tensile specimens.
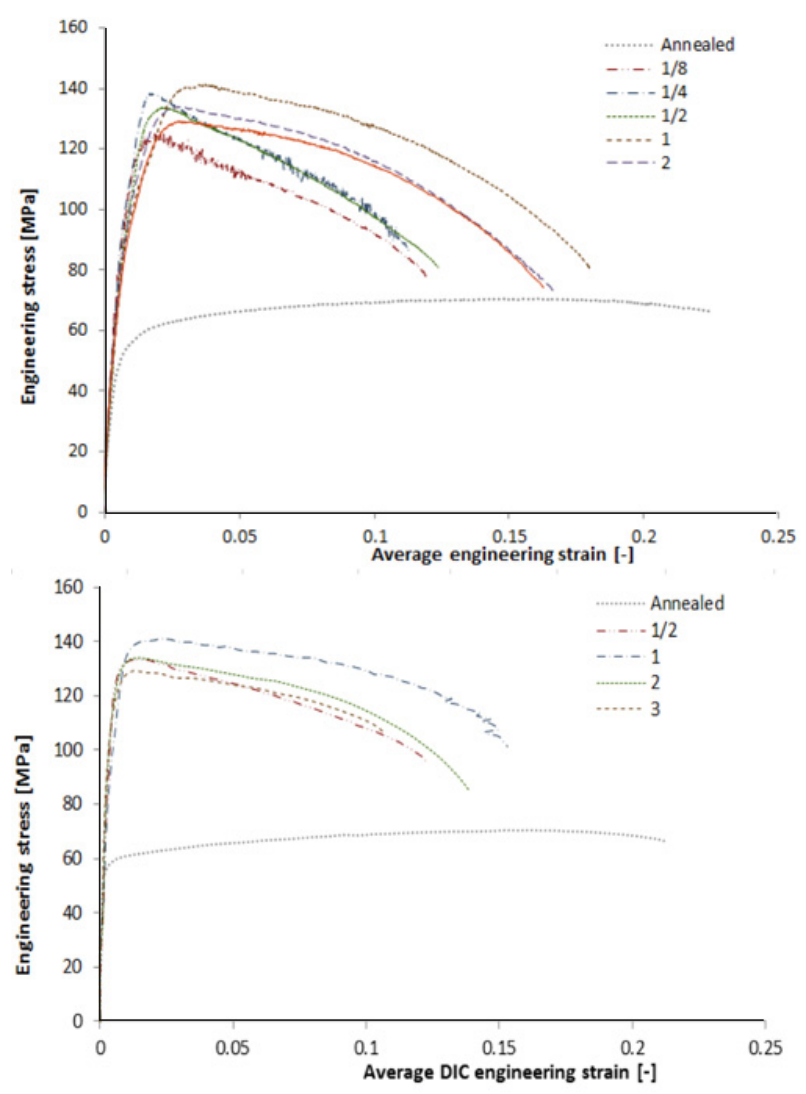

Figure 5. Static stress-strain curves of annealed and HPT processed material. The strain is calculated from the clamp displacement (top) or by DIC (bottom).

\subsection{Static and dynamic tensile curves}

In Fig. 5 the static tensile curves for the annealed and HPT samples are represented. In the top figure the strain values are averaged values obtained by dividing the clamp displacements by the initial gage length.

In Fig. 6 images of a deforming sample are given together with the results of DIC by which local strain values are obtained. The strain distribution is clearly nonuniform: in the top picture strains vary from 1 to $15 \%$, in the bottom picture from 1 to $47 \%$. Unfortunately, at high deformations, paint flaked off in the neck region and DIC strain values become unreliable.

In the bottom Fig. 5, curves are presented for the tests for which reliable DIC strain values are obtained. Before necking, the average strains obtained from the clamp displacements (top Fig. 5) overestimate the average strains in the gage section (bottom Fig. 5). For the dimensions of the tensile samples used here, deformation of the transitions zones of the sample, i.e. the shoulders, is limited [10]; the differences observed are mainly attributed to the slip in the clamps and a limited stiffness of the tensile device.

HPT processing has a pronounced influence on both the stress and elongation values. In the annealed sample the deformation is uniform up to a strain of $15 \%$. In the HPT samples on the other hand, very soon after the onset of plastic deformation the curves reach a maximum and necking sets in. For the tensile tests represented in 


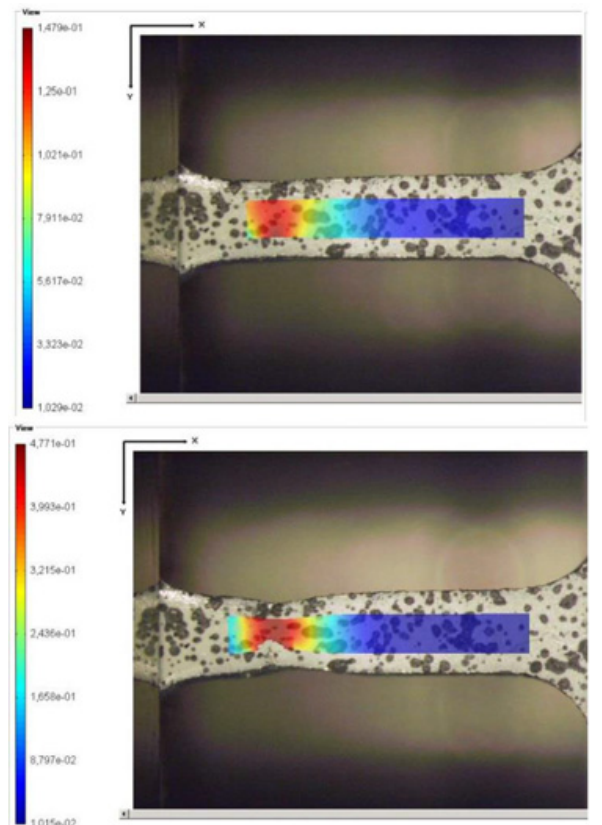

Figure 6. Sample during deformation with axial strain obtained by DIC processing (using MatchID ${ }^{\mathrm{TM}}$ ) of the speckle pattern applied to the sample.

the bottom of Fig. 5, uniform elongations between 1.2\% and $2.2 \%$ are found. A higher HPT deformation indeed results in lower uniform strains. From the tensile results a considerable increase in the ultimate tensile strength (UTS) of the material, even at lowest HPT deformation, is obtained compared with annealed samples.

The annealed sample has a tensile strength of $70 \mathrm{MPa}$. The strength increases significantly to a value of $125 \mathrm{MPa}$ for the $1 / 8$ rotated sample, to $138 \mathrm{MPa}$ for the $1 / 4$ sample. For higher HPT deformations, the changes in maximum stress level cannot directly be related with the applied HPT deformation and/or obtained grain refinement. Indeed, although no differences in grain size exist between the 2 and 3 rotations samples, the maximum stress of the sample of 3 rotations is lower than that of the 2 rotations sample. This might be attributed to the value of the cross section used to calculate the strain from the measured force. Here, the dimensions of the central section are used. However, in the HPT processed samples, a higher reduction of the sample thickness is obtained away from the centre, so this will result in an underestimation of the actual stress. The differences in thickness over the gage length are more pronounced for high levels of HPT deformation. This is in line with the observation that for higher HPT deformations, the strain localisation preceding fracture, occurs further away from the centre of the gage section.

In Fig. 7 next to static tensile curves, dynamic curves are given obtained by the split Hopkinson bar setup. The average strain rate during plastic yielding is $4400 / \mathrm{s}$. When the static curves are compared with the dynamic curves, some trends can be observed. The effect of the strain rate on the onset of yielding and uniform deformation lies within the experimental scatter. HPT processing does not seem the affect the materials strain rate sensitivity. However, once necking sets in, the stress decreases more

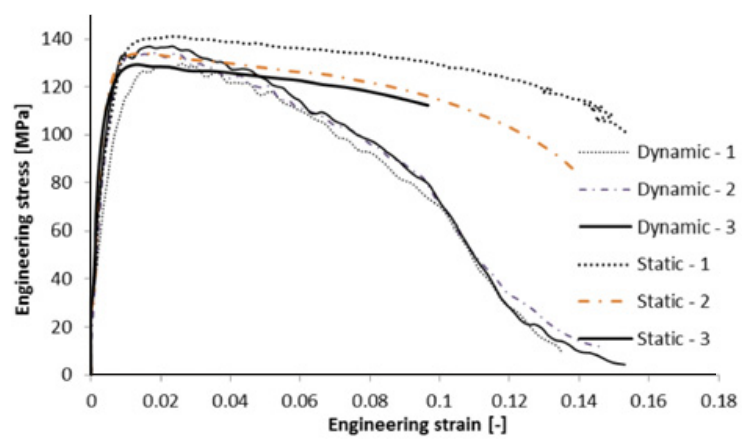

Figure 7. Static and dynamic stress-strain curves for the HPT samples subjected to 1,2 and 3 rotations.
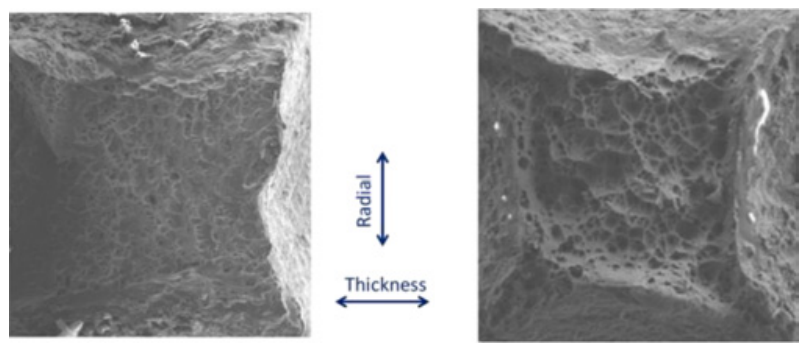

Figure 8. Fracture surface of a sample subjected to $1 / 2$ rotations in HPT, corresponding with an equivalent strain of 5.4, after a static (left) and dynamic (right) tensile test.

rapidly at higher strain rates. This might be attributed to thermal softening of the material due to adiabatic heating of the samples in the dynamic tests.

\subsection{Post mortem analysis}

In all samples a cup and cone fracture is observed with clear dimples typical for ductile fracture preceded by void nucleation, growth and coalescence. Fracture surfaces of the static and dynamic tensile samples are observed using scanning electron microscopy (SEM). In SEM images of the fracture surfaces equiaxed dimples around the centre and parabolic shear dimples in the narrow shear lips close to the borders are present, as can be seen in Fig. 8 for a sample subjected to $1 / 2$ rotations in HPT after a static (left) and dynamic (right) tensile test.

In Fig. 9 the engineering radial and thickness strain as measured in the symmetry planes in the fracture surface are represented as a function of applied strain in the HPT process. The radial strain is in all static (Fig. 9, top) and dynamic tests (Fig. 9, bottom) higher than the thickness strain, although there is some evolution as a function of applied deformation. The anisotropy as observed in the fracture surface, reaches its highest value at a HPT von Mises strain of 2.7. However, it should be mentioned that the strain values at higher deformations, are less accurate because of the thickness variation over the gage length of the sample.

From Fig. 9 it is also clear that, although the uniform deformation of the sample is limited, at fracture high deformations are obtained. The lowest values of the radial and thickness strain are obtained in the dynamic experiments; however, their value is well above $50 \%$. Consequently, although the tensile curves suggest a low 


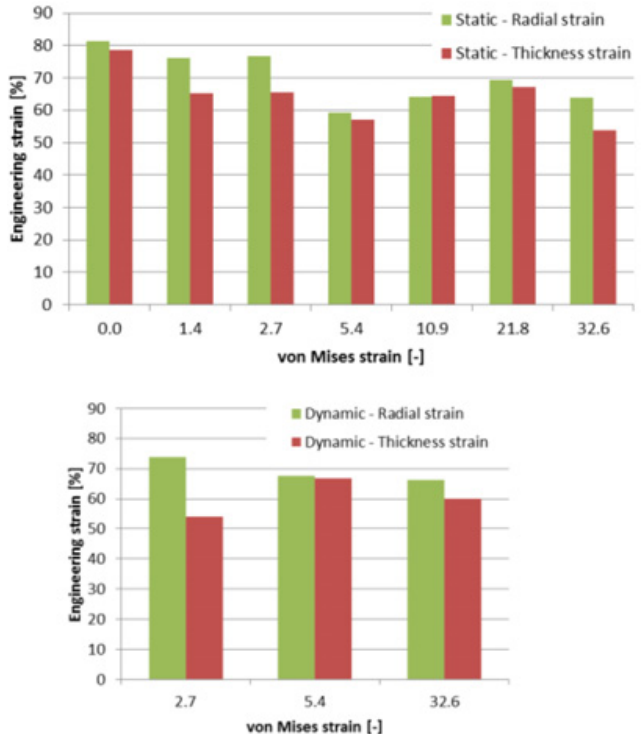

Figure 9. Fracture strain as a function of strain applied by HPT in the radial and thickness direction measured from SEM images of the fracture surfaces for the static (top) and dynamic (bottom) samples.

tensile ductility of the HPT processed aluminium, the material is intrinsically ductile. The low uniform tensile deformation is the result of a geometrical instability.

For low HPT deformations, the dimples in the fracture surface are approximately 12 microns, which is much smaller than the grain size. Independent of the strain rate, the dimple size decreases with increasing HPT strain, until it saturates at $\approx 9$ micron, approximately nine times the grain size for samples subjected to an equivalent strain higher than 10.

\section{Conclusions}

In present study, the static and dynamic tensile behaviour of commercially pure aluminium processed by HPT is studied. The deformation applied to the samples by HPT processing prior to tensile testing results in a significant grain refinement: from a grain size of 300 micron in the annealed state to a saturation size of $\sim 1 \mu \mathrm{m}$ for equivalent strains higher than 15 . The grain size reduction results in a strength increase of the order of 2 , in both the static and dynamic tests. For the commercially pure aluminium considered here, the influence of the strain rate on the onset of plastic deformation in the samples and the uniform elongation is found to be negligible. At higher strains, beyond necking, the stress drops more rapidly in the dynamically loaded samples, which might be attributed to thermal softening. Observation of the fracture surfaces shows that, independent of deformation level or strain rate, a ductile cup and cone fracture is obtained. The dimple size decreases with increasing HPT deformation to reach a saturation level when also the grain size saturates. Measurement of the strains in the fracture surface shows a slight difference in strain along the thickness and radial direction of the samples. The anisotropy seems to be more pronounced for low levels of deformation. The fracture strains are lower in the dynamic tests, although also here values well above $50 \%$ are found. Consequently, although the tensile curves suggest a low tensile ductility of the HPT processed aluminium, the HPT processed pure aluminium is intrinsically ductile.

The authors would like to acknowledge the Interuniversity Attraction Poles Program (IUAP) of the Federal Science Policy of Belgium and the partners of IUAP-VII-project P7/21 "Multiscale mechanics of interface dominated materials".

Also the help of Dr. Farideh Hajy Akbary of the Department of Materials Science and Engineering at TUDelft (The Netherlands) for carrying out the static tensile experiments is gratefully acknowledged.

\section{References}

[1] V. Segal, Mater. Sci. Eng. A 197 (1995)

[2] A. Zhilyaev, T. Langdon, Progr. Mat. Sci. 53 (2008)

[3] T. Lowe, R. Valiev, Nanostruct. Mater. 10 (1998)

[4] Y. Sait, H. Utsunomia, N. Tsuji, T. Sakai, Acta Mater. 47 (1999)

[5] E. Ma, J. Mat. Eng. Perf. 14 (2005)

[6] G. Dieter, Mechanical Metallurgy. $8^{\text {th }}$ ed. New York, McGrw-Hill (1998)

[7] Q. Wei, S. Cheng, K.T. Ramesh, E. Ma, MSEA 381 (2004)

[8] Y. Wang E. Ma, Acta Mat. 52 (2004)

[9] Y.M. Wang, E. Ma, MSEA 375-377 (2004)

[10] P. Verleysen, J. Degrieck, T. Verstraete, J. Van Slycken, Exp. Mech. 48 (2008) 